\title{
Evaluation of the donor site after the median forehead flap
}

\author{
June Seok Choi ${ }^{1}$, Yong Chan Bae ${ }^{1,2}$, Soo Bong $\mathrm{Nam}^{1}$, Seong Hwan Bae ${ }^{1}$, Geon Woo Kim ${ }^{1}$ \\ ${ }^{1}$ Department of Plastic and Reconstructive Surgery, Pusan National University School of Medicine, Busan; ${ }^{2}$ Biomedical Research Institute, \\ Pusan National University Hospital, Busan, Korea
}

Background Forehead flaps are useful for facial reconstruction. Studies of these flaps have mostly focused on the results of the reconstruction. However, due to the scarring and changes on the forehead caused by the median forehead flap (MFF), surgeons may be reluctant to perform this flap. Research into the donor site is needed for practical purposes.

Methods We examined 42 patients who underwent an MFF at Pusan National University Hospital from 1996 to 2016. Based on a retrospective chart review, we examined the occurrence of complications. We also evaluated scars on the forehead using the Vancouver Scar Scale (VSS) and assessed changes in the eyebrow position of 22 patients.

Results No complications occurred in the 42 patients. The mean VSS score of the 22 patients was $2.8 \pm 0.79$. The ratio of the height of the eyebrow on each side to the distance between the medial canthi increased postoperatively, meaning that both the left and right brows were elevated slightly $(P=0.026$ and $P=0.014)$. However, the symmetry between the left and right sides did not change $(P=0.979)$. The ratio of the interbrow distance to the distance between the medial canthi decreased slightly, meaning that the interbrow distance narrowed mildly $(\mathrm{P}<0.001)$. Moreover, there were no noticeable changes in the brow position as seen in a photo overlay.

Conclusions There were no notable complications in the forehead. Forehead scarring was acceptable. No change in brow symmetry was observed via photographic measurements and a photo overlay. Therefore, we propose that the MFF is a useful choice for minimizing scarring or deformation of the donor site.

Keywords Forehead / Surgical flaps / Cicatrix / Eyebrows
Correspondence: Yong Chan Bae Department of Plastic and

Reconstructive Surgery, Biomedical Research Institute, Pusan National University Hospital, Pusan National University School of Medicine, 179 Gudeok-ro, Seo-gu, Busan 49241, Korea

Tel: $+82-51-240-7273$

Fax: +82-51-243-9405

E-mail: baeyc2@hanmail.net

This work was supported by a 2-year research grant from Pusan National University.

Received: 8 Aug $2017 \bullet$ Revised: 15 Mar $2018 \bullet$ Accepted: 5 Apr 2018

pISSN: 2234-6163 • elSSN: 2234-6171 • https://doi.org/10.5999/aps.2017.01277• Arch Plast Surg 2018;45:259-265

\section{INTRODUCTION}

Forehead flaps are useful for facial reconstruction, particularly for the nose, and also for various areas such as the eyelid or canthus. They are useful because their color and texture are similar to those of the areas to be reconstructed and because they have good vascularity. Moreover, they can be designed in diverse shapes to fit various needs [1-5]. However, in some cases, due to the scar on the forehead caused by the flap, surgeons may be reluctant to perform this flap. Studies of forehead flaps have mostly focused on the results of the reconstruction, not on the donor site (forehead), despite the need for such research. Prior studies either did not show the forehead at all or sometimes covered the donor site with scalp hair [2]. 
In this study, the authors examined the donor sites on the forehead among patients who underwent reconstruction using a median forehead flap. The occurrence of complications, scars, and changes in the position of the eyebrows were evaluated. Therefore, we intended to investigate whether problems occurred at the donor site after the forehead flap and to provide quantitative information about the donor site.

\section{METHODS}

We examined 42 patients who underwent a forehead flap procedure at Pusan National University Hospital from October 1996 to January 2016 and whose medical records were available. We conducted this study after receiving approval from the Institutional Review Board (IRB No. H-1801-009-063). Informed written consent was obtained from all patients.

Among the patients, 19 were men and 23 were women. Their mean age was 61.8 years old, ranging from 18 to 79 years. The mean postoperative follow-up period was 36 months, ranging from 12 months to 16 years. The most common cause of the facial defect was basal cell carcinoma removal (32 cases), followed by squamous cell carcinoma removal ( 4 cases), dermatofibrosarcoma protuberans removal ( 2 cases), and mucinous carcinoma removal (1 case). Two defects were caused by an injury, and one defect by an inflammatory deformity. In 33 cases, the defect was located in the nose, followed by eight cases of defects around the eyes, and one case in the suprabrow region. The defects in the nose were categorized into the alar region (20 cases), dorsum ( 6 cases), side wall ( 4 cases), and tip ( 3 cases). If a defect affected two or more regions, the most dominant area was chosen for the classification. The defects around the eyes were located in the medial canthal region (6 cases), lateral canthal region ( 1 case), and lower eyelid ( 2 cases).

\section{Surgical technique}

The median forehead flap was designed to have a base width of $2 \mathrm{~cm}$, with the glabella region as a pivot point. This design choice was made because a width of more than $2 \mathrm{~cm}$ is very likely to cause problems at the donor site. The flap was designed to be straight or curved on an individualized basis to ensure that the hair, position, size, and shape of the defect would be covered. In accordance with the design, a paper pattern was created in the surgical field, and was virtually pivoted to check that it would cover the defect. The flap was elevated in the sub-periosteal layer. Following this elevation, however, we frequently performed additional selective flap thinning to match the native skin of the nose. The plane continued toward the medial brow region. This dissection must be done meticulously to avoid in- juring the pedicle and muscle.

All donor sites were closed primarily with wide undermining. Additionally, the flap was observed carefully, with the goal of minimizing pressure on the subcutaneous pedicle, as flap congestion is probably the greatest risk of this procedure.

\section{Complications}

Based on a retrospective review of the records of the 42 patients who underwent a median forehead flap procedure, we examined the occurrence of the following complications: necrosis on the flap and forehead, hematoma, and infection.

\section{Donor site analysis}

We also evaluated the donor sites in the 22 patients whose donor site was closed primarily. They were followed up for 1 year or longer after the procedure, who were able to be measured using a scar scale, and whose photos from before and after surgery were available. Patients who underwent surgery of the brow or experienced facial palsy were excluded.

\section{Scar scale}

Scarring at the donor site of the forehead flap 1 year or more after surgery was evaluated. Using the Vancouver Scar Scale (VSS), the pigmentation, vascularity, pliability, and height of the donor site were assessed by a gross examination. The VSS yields scores between 0 and 14, with higher scores indicating a more severe scar.

\section{Brow position}

To evaluate changes in the brow position, we compared changes in the height of the left and right eyebrows before and after surgery. We also measured changes in the symmetry of the brows by comparing the left and right brows before and after surgery.

Fig. 1. Measurements made from the photos

$A$, distance between medial canthi; $B$, distance from medial canthus

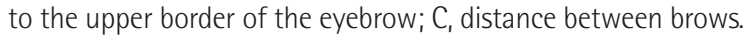

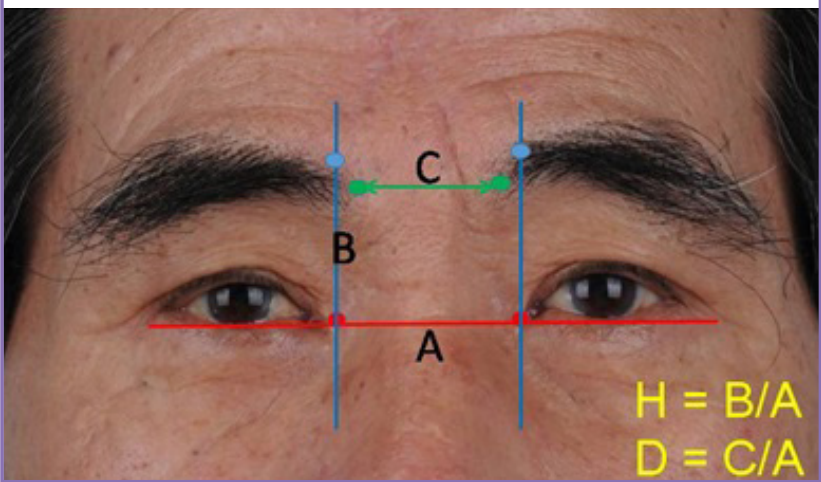


To make measurements from photos, an imaginary line connecting the medial canthus of each eye was set as a horizontal axis. Two lines were drawn perpendicularly from the medial canthus of both sides until the lines met the upper border of each brow (B). Then, the ratio $(\mathrm{H})$ of the distance between each medial canthus and the point at the upper border of each brow (B) to the distance between both medial canthi (A) was measured (Fig. 1).

To evaluate changes in the position of the right eyebrow, the preoperative value of the right side (pre- $\mathrm{RH}$ ) and the postoperative value of the right side (post-RH) were compared. To evaluate changes in the position of the left eyebrow, the preoperative value of the left side (pre-LH) and postoperative value of the left side (post-LH) were compared. To evaluate the preoperative symmetry of the eyebrows, the pre- $\mathrm{LH}$ and pre- $\mathrm{RH}$ values were compared. To evaluate the postoperative symmetry of the eyebrows, the post-LH and post-RH values were compared. To evaluate the change in the symmetry of the eyebrows, the difference between the pre- $\mathrm{LH}$ and pre- $\mathrm{RH}$ values was compared to the difference between the post- $\mathrm{LH}$ and post-RH values. To examine changes in the interbrow distance, the distance between the brows (C) and the ratio of $\mathrm{C}$ to $\mathrm{A}(\mathrm{D})$ were calculated.

\section{Overlapping photos}

We overlaid the preoperative and postoperative photos to perform intuitive evaluations. In the preoperative photo of a given patient, an imaginary line connecting the medial canthus of each eye was set as the horizontal axis. The same was done in the postoperative photo. The size of the two photos was adjusted to be equal. Then, one photo was overlaid over the other. The transparency of the photos was adjusted to ensure that both photos were visible. Finally, five plastic surgeons evaluated the photos using the following scale: (1) severe, (2) moderate, (3) mild, (4) minimal, (5) no change.

\section{Statistical analysis}

For statistical analysis, nonparametric testing using the Wilcoxon test was conducted using IBM SPSS version 23 (IBM Corp.,

\section{Table 1. Vancouver Scar Scale results}

\begin{tabular}{|ll|}
\hline & Value \\
\hline Pigmentation & $0.5 \pm 0.51(0-1)$ \\
\hline Pliability & $1.5 \pm 0.67(0-2)$ \\
Height & $0.3 \pm 0.48(0-1)$ \\
Vascularity & $0.5 \pm 0.57(0-1)$ \\
Total score & $2.8 \pm 0.79(1-3)$ \\
\hline Values are presented mean \pm SD (range). \\
The donor site (forehead) showed acceptable scarring. \\
\hline
\end{tabular}

Armonk, NY, USA). P-values $<0.05$ were considered to indicate statistical significance.

\section{RESULTS}

\section{Complications}

Among the 42 patients who underwent a median forehead flap procedure, complications such as necrosis, hematoma, infection, and wound dehiscence did not occur. In two cases, wound dehiscence on the forehead occurred after the first operation. During the second flap division operation, marginal resection and primary suturing were performed, and the wound healed without further problems.

\section{Donor site analysis}

Scar scale

On the VSS, pigmentation was scored as $0.5 \pm 0.51$, pliability was scored at $1.5 \pm 0.67$, height was scored as $0.3 \pm 0.48$, vascularity was scored as $0.5 \pm 0.57$, and the total score was $2.8 \pm 0.79$ (Table 1).

\section{Brow position}

The ratio of the height of the left brow to the distance between the medial canthi increased postoperatively; the left brow was slightly elevated $(\mathrm{P}=0.026)$, and the right brow was also elevated mildly after the procedure $(\mathrm{P}=0.014)$ (Table 2$)$.

Before the procedure, the position of the left brow was found to be slightly higher than the right brow $(\mathrm{P}=0.003)$. Postopera-

\section{Table 2. Changes in brow height}

\begin{tabular}{|c|c|c|c|c|}
\hline & Preoperative & Postoperative & Difference & P-value \\
\hline Left H & & & & \\
\hline Right H & & & & \\
\hline \multicolumn{5}{|c|}{$\begin{array}{l}\text { Values are presented mean } \pm S D \text {. } \\
\text { Left brow was slightly elevated }(P=0.026) \text {, and the right brow was also elevated } \\
\text { mildly after the procedure }(P=0.014) \text {. H was defined as the distance from the } \\
\text { medial canthus to the upper border of the eyebrow divided by the distance } \\
\text { between the medial canthi. }\end{array}$} \\
\hline
\end{tabular}

Table 3. Differences between the left and right brows

\begin{tabular}{|c|c|c|c|c|}
\hline & Left $H$ & Right H & Difference & P-value \\
\hline Preoperative & $0.73 \pm 0.13$ & $0.70=$ & 0.04 & 0.003 \\
\hline Postoperative & $0.77 \pm 0.14$ & $0.74 \pm$ & & \\
\hline \multicolumn{5}{|c|}{$\begin{array}{l}\text { Values are presented mean } \pm S D \text {. } \\
\text { Before the procedure, the position of the left brow was found to be slightly higher } \\
\text { than the right brow }(P=0.003) \text {. Postoperatively, the left brow was likewise slightly } \\
\text { higher than the right brow. H was defined as the distance from the medial } \\
\text { canthus to the upper border of the eyebrow divided by the distance between the } \\
\text { medial canthi. }\end{array}$} \\
\hline
\end{tabular}


tively, the left brow was likewise slightly higher than the right brow $(\mathrm{P}=0.013)$ (Table 3).

When the gap between the pre- $\mathrm{LH}$ and pre- $\mathrm{RH}$ values was compared with that between the post- $\mathrm{LH}$ and post-RH values, the difference was minimal and not statistically significant $(P=0.979)$. Therefore, it was confirmed that the symmetry of the eyebrows did not change after the procedure (Table 4).

The ratio of the interbrow distance to the distance between the medial canthus decreased slightly, showing that the interbrow distance narrowed mildly $(\mathrm{P}<0.001)$ (Table 5).

\section{Overlapping photos}

When we evaluated changes in the position of the brows via a photo overlay, the average score was $4.67 \pm 0.14$, between minimal change and no change.

\section{Cases}

Case 1

A 70-year-old male was diagnosed with basal cell carcinoma in the alar region. He underwent Mohs micrographic surgery (MMS) and a median forehead flap procedure for reconstruc-

Table 4. Changes in brow symmetry

\begin{tabular}{|c|c|c|c|c|}
\hline & Preoperative & Postoperative & Difference & P-value \\
\hline $\begin{array}{l}\text { Difference } \\
\text { between left } \\
\text { and right H }\end{array}$ & $0.03 \pm 0.07$ & $0.04 \pm 0.07$ & $0.01 \pm 0.02$ & 0.979 \\
\hline
\end{tabular}

tion. The postoperative evaluation was conducted 6 years after the procedure. The VSS score was 3 . No change in brow symmetry was observed (Fig. 2).

\section{Case 2}

A 38-year-old female was diagnosed with dermatofibrosarcoma protuberans in the suprabrow region. She underwent MMS and a median forehead flap procedure for reconstruction. The postoperative evaluation was conducted 16 years after the procedure. The VSS score was 2 . No change in the brow symmetry was observed (Fig. 3).

\section{Case 3}

A 64-year-old male was diagnosed with basal cell carcinoma of the lower eyelid. He underwent MMS and a median forehead flap procedure for reconstruction. The postoperative evaluation was conducted 5 years after the procedure. The VSS score was 3. No change in the brow symmetry was observed (Fig. 4).

\section{DISCUSSION}

Forehead flaps are useful for facial reconstruction, because of their color, texture, size, reach, vascularity, lining applications,

\section{Table 5. Changes in the interbrow distance}

\begin{tabular}{|c|c|c|c|c|}
\hline & Preoperative & Postoperative & Difference & P-value \\
\hline D & $0.70 \pm 0.14$ & $0.66 \pm 0.15$ & $-0.04 \pm 0.05$ & $<0.001$ \\
\hline
\end{tabular}

\section{Fig. 2. Case 1}

A 70-year-old male who was diagnosed with basal cell carcinoma in the alar region. (A) After Mohs micrographic surgery. (B) Median forehead flap state. (C) Postoperative photo obtained 6 years after reconstruction, with a Vancouver Scar Scale score of 3. (D) Overlapping photos: no change in brow symmetry.
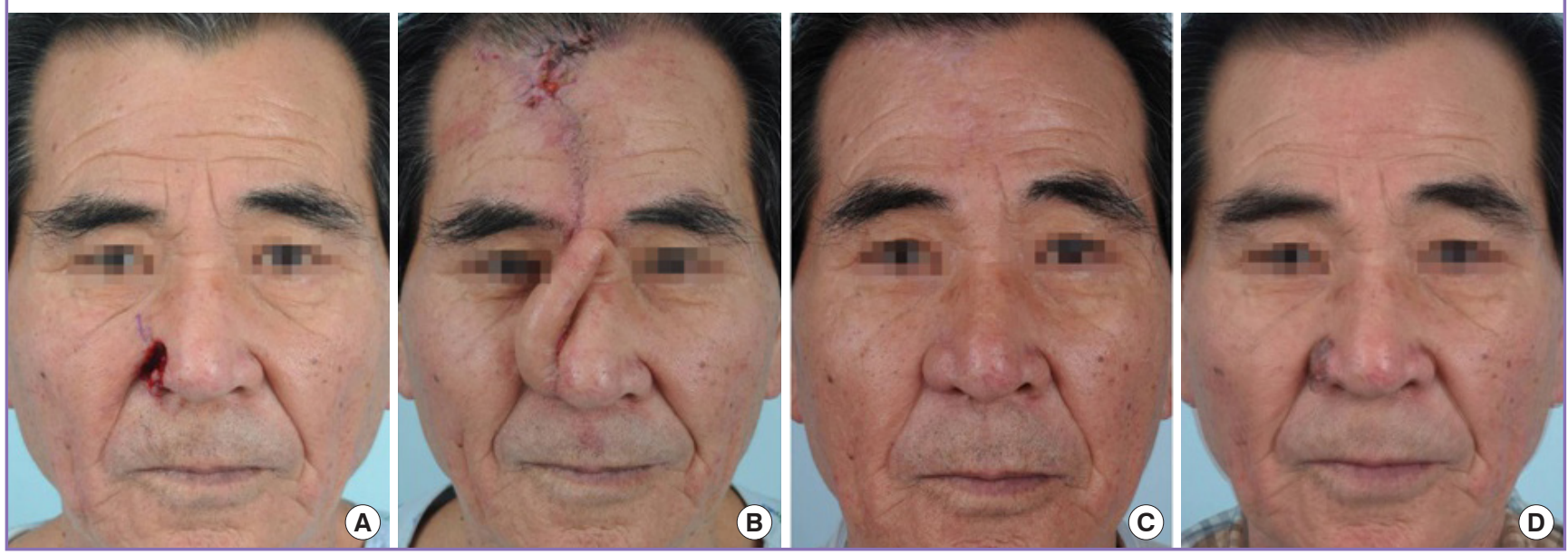
and forgiving donor site. Paramedian forehead flaps have advantages over median forehead flaps in terms of flap size and the size and length of the vessels [3].

This study provides meaningful results because it reports findings from a large sample of 42 patients' donor sites, instead of the recipient sites, which have traditionally been used to assess the results of reconstruction.

No severe complications, such as flap necrosis, occurred after median forehead flap procedures. This is speculated to have be due to the fact that the flaps were supplied by the paired supratrochlear artery $[6,7]$.

Scars on the donor site were evaluated using the VSS. This scale is the most widely used in clinical practice for measuring scars. The mean total score of the VSS was 2.8. Moreover, no complaints about scarring were reported by the patients. Overall, the extent of scarring on the donor site of the median fore-

\section{Fig. 3. Case 2}

A 38-year-old female who was diagnosed with dermatofibrosarcoma protuberans in the suprabrow region. (A) After Mohs micrographic surgery. (B) Median forehead flap state. (C) Postoperative photo obtained 16 years after reconstruction, with a Vancouver Scar Scale score of 2. (D) Overlapping photos: no change in brow symmetry.
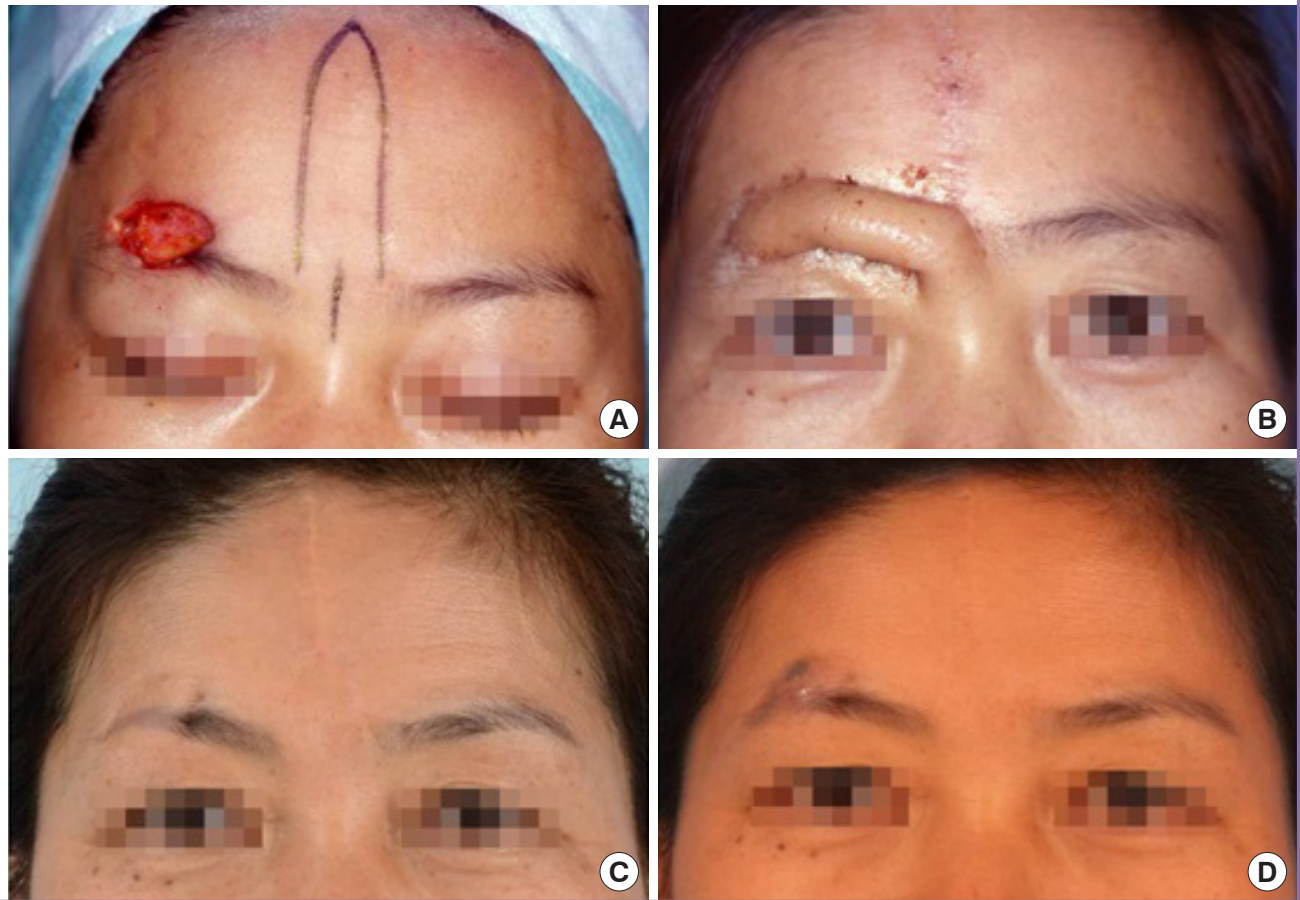

\section{Fig. 4. Case 3}

A 64-year-old male who was diagnosed with basal cell carcinoma of the lower eyelid. (A) After Mohs micrographic surgery. (B) Median forehead flap state. (C) Postoperative photo: 5 years after reconstruction, with a Vancouver Scar Scale score of 3. (D) Overlapping photos: no change in brow symmetry.
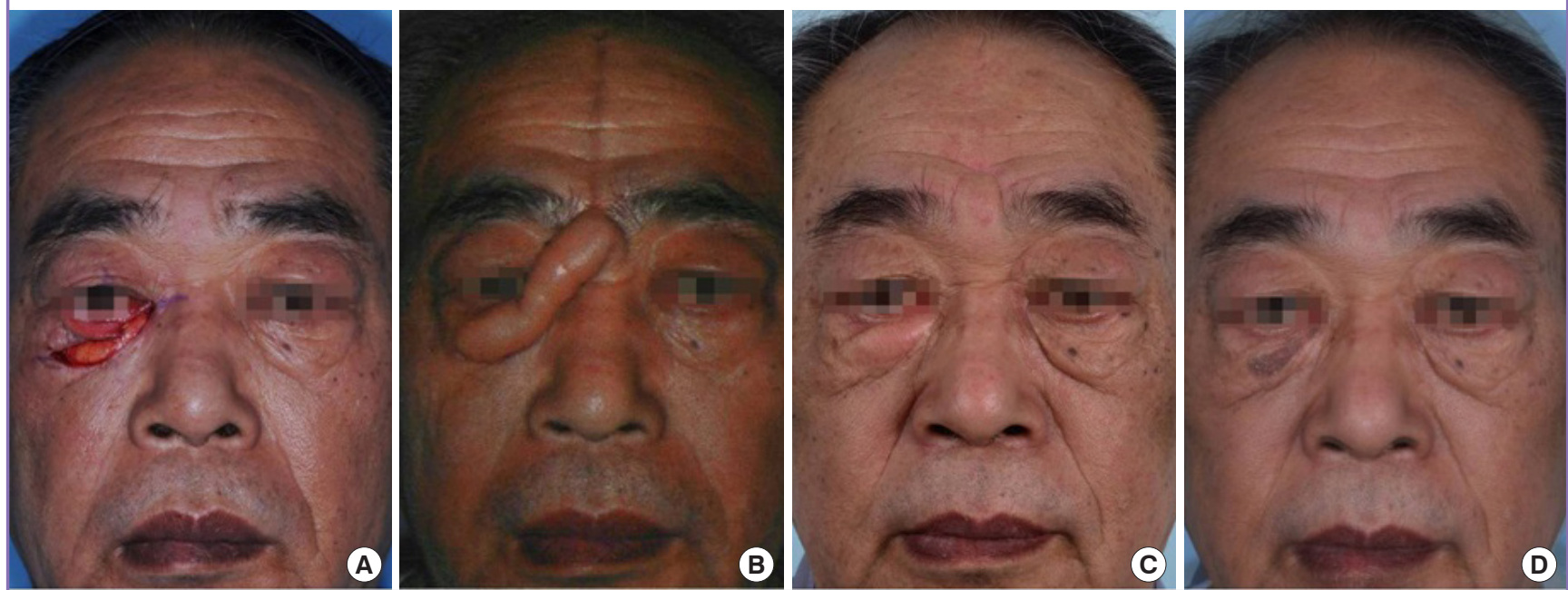
head flap can be considered acceptable [8].

Measuring photos of the donor sites is very important. In this study, a horizontal reference line was set as an imaginary line connecting the medial canthi of both eyes. This line is relatively unaffected over time and serves as an anatomical landmark.

A vertical reference line was set by drawing a perpendicular line from each medial canthus to the upper border of each brow. The distance between the horizontal reference line and the border of the upper brow was measured. To avoid errors caused by different photo sizes, we did not measure absolute distances for brow height. Instead, we used the ratio of the interbrow distance to the distance between the medial canthi [9-12].

According to the assessments made using the photos, compared to the preoperative position, the overall height of the brows after surgery was elevated and the interbrow distance was narrowed. We propose that these changes were due to contracture in the wound healing process after the detachment caused by surgery. However, no changes in brow symmetry, which can occur after paramedian forehead flap procedures, were seen.

Finally, in an intuitive assessment of the change in the position of the brows via overlaid photos, no change in the eyebrow position was observed. This indirectly suggests that the small difference shown in previous measurements was not visible to the naked eye.

The primary limitation of this study is that it only included patients who underwent median forehead flap surgery. It is necessary to study and compare the donor sites of patients who underwent operations using different forehead flaps. Another limitation is that the median forehead flap was selectively offered. We limited the flap base width to $2 \mathrm{~cm}$ to ensure primary closure of the donor site, making it problematic to use this flap for large defects. Therefore, we resolved remaining areas of the defects using additional methods (nasolabial flaps or skin grafts).

In conclusion, this study showed that there were no complications in patients who underwent a median forehead flap, and that the donor site (forehead) showed acceptable scarring. In photographic measurements, the height of the brows was elevated slightly and the interbrow distance was narrowed postoperatively; there was no change in symmetry. Moreover, in an intuitive assessment of the change in the position of the brows via overlaid photos, no change in the eyebrow position was observed, unlike the results obtained through photographic measurements. Therefore, we found that there were no problems with the donor site of the median forehead flap and propose that the median forehead flap can be a useful choice for minimizing scarring or deformation of the donor site.

\section{NOTES}

\section{Conflict of interest}

No potential conflict of interest relevant to this article was reported.

\section{Ethical approval}

The study was approved by the Institutional Review Board of Pusan National University Hospital (IRB No. H-1801-009063) and performed in accordance with the principles of the Declaration of Helsinki. Written informed consents were obtained.

\section{Patient consent}

The patients provided written informed consent for the publication and the use of their images.

\section{REFERENCES}

1. Menick FJ. Nasal reconstruction: forehead flap. Plast Reconstr Surg 2014;113:100e-111e.

2. Price DL, Sherris DA, Bartley GB, et al. Forehead flap periorbital reconstruction. Arch Facial Plast Surg 2004;6:222-7.

3. Menick FJ. Aesthetic nasal reconstruction. In: Eduardo DR, editor. Plastic surgery. 3th ed. Elsevier; 2012. p.134-86.

4. Park SS. The single-stage forehead flap in nasal reconstruction: an alternative with advantages. Arch Facial Plast Surg 2002;4:32-6.

5. McCarthy JG, Lorenc ZP, Cutting C, et al. The median forehead flap revisited: the blood supply. Plast Reconstr Surg 1985;76:866-9.

6. Reece EM, Schaverien M, Rohrich RJ. The paramedian forehead flap: a dynamic anatomical vascular study verifying safety and clinical implications. Plast Reconstr Surg 2008; 121:1956-63.

7. Skaria AM. The median forehead flap reviewed: a histologic study on vascular anatomy. Eur Arch Otorhinolaryngol 2015;272:1231-7.

8. Bae SH, Bae YC. Analysis of frequency of use of different scar assessment scales based on the scar condition and treatment method. Arch Plast Surg 2014;41:111-5.

9. Kim BP, Goode RL, Newman JP. Brow elevation ratio: a new method of brow analysis. Arch Facial Plast Surg 2009; 11:34-9.

10. Kokubo K, Katori N, Hayashi K, et al. Evaluation of the eyebrow position after levator resection. J Plast Reconstr Aesthet Surg 2017;70:85-90.

11. Verduijn PS, Selles RW, Mureau MA. A simple, reliable, and 
validated method for measuring brow position. Ann Plast Surg 2014;73:81-5.

12. Macdonald KI, Mendez AI, Hart RD, et al. Eyelid and brow asymmetry in patients evaluated for upper lid blepharoplasty.J Otolaryngol Head Neck Surg 2014;43:36. 